\title{
Epidemiología del Hábito de Beber en Adolescentes
}

\author{
Dr. Armando Nader $\mathrm{N}^{1}{ }^{1}$; Dr. Julio Pallavíìi $G_{.}{ }^{2}$; Dral. Adula Legareta $\mathrm{P}^{3}{ }^{3}$; Dr. Jorge Mahaluf $Z_{-}{ }^{4}$; \\ Sr. Fránciseo Cumsille G. ${ }^{5}$; Sr. Claudio Silva Z. ${ }^{6}$; Sr. Claudio Vila $Z_{.}{ }^{7}$.
}

\section{Epidemiology of Drinking Habits Among Adolescents}

\begin{abstract}
The epidemiology of alcoholism und other dritking habits among adolescents lotween 15 and 19 years old from Santiago (Chile) were studicd. The sample was made of 456 youngsters, and it was obtained from the general population through an aleatory systematic method. $A$ clinital diagnosis wis done from the results of an individual standarized interview. The prevalence rate of akcoholism reached $9.2 \%$ being higher among males with a female/male rate of $1 / 4(p<0.005)$. There were more alcoholics in the lower sacial $(20.8 \% \mathrm{p}<0.005)$ and cducational levels $(15.6 \%, p<0.01)$ and among youngsters that werc not studying $(14.3 \% \mathrm{p}<0.025)$. $\wedge \mathrm{n}$ epidemiologic profile of the youngsterw with the highest risk wis established: man, adolescent, low suciat level, poor education, not studying neither working.

( Key words: Adolescent. Alcoholism. Drinking habits, Educational level. School. Work.).
\end{abstract}

El enfoque clínico del Alcoholismo ${ }^{1}$, ha abierto nuevos caminos de investigación, tanto a nivel individual como poblacional. Basados en dicho enfoque, en 1982 realizamos una inyestigación clínica y epideıniológica sobre el alcoholismo entendido como apetencia patológica, en la población de 15 años y más, de Santiago ${ }^{2}$, cuya tasa de prevalencia de alcoholismo alcanzó a $12,6 \%$. Ya entonces pareció importante caracterizar las diferentes formas de beber en cl adolescente, para actualizar las tasas de prevalencia tomando en cuenta los nuevos puntos de vista clínicos, y para averiguar su relación con varia. bles bio y socio-demográficas que hasta la fecha no se habían estudiado en la población general.

Los estudios epidemiológicos nacionales se han efectuado principalmente en adolescentes escolares pero las definiciones y muestras empleadas son tan divergentes, que hacen imposible su comparación. La revisión de las publicaciones

(*) Este estudio corresponate a uno de los trabajos de la "Investigación Clínica y lipidemiológiét sobre Alcoholismo y otras Apetencias", en la que participan: el Dpto. de Psiquiatría y Salud Mental, Div. Cs. Ms. Norte y la Escucla de Salud Pública, Yacultad de Medicina, Lniversidad de Chile: Laboratorio de Estadístiea de la Universidad de Santiago de Chile y el Instituto Nacional de Fstadística.

(1) Profesor Auxiliar de Psiquiatría.

(2) Profesor Asociado de Psiquiatría.

(3) Profesor Títulat de Biocstadística.

(4) Ayudante Primero de Psiquiatria.

(5) Master en Estadistica Matemática.

(6) Doctor en Bioestadistica.

(7) Demógrato. Profesor de Matemáticas. disponibles muestran cif ras de bebedores anormales que van desde 0.8 a $13 \%$, utilizando para el diagnóstico đe dicha condición criterios cuanti. tativos, tales como la frecuencia de la ingesta y embriaguez $z^{3}-4-5-6-7$. Jdénticas dificuliades se presentan también con la información del extranjero ${ }^{8 \cdot 9 \cdot 10}$.

Si bien los estudios entre escolares son importantes. se deben tomar en cuenta, además. los adolescentes que no estudian, ya sca que se encuentren trabajando o cesintes, pues el alcoholismo se da también en ellos y para medir correctamente el problema es nccesario incluirlos.

Sin embargo los trabajos chilenos de prevalencia en la población general son pocos. Destaca el de Horwitz que en 1958 encontró entre los audolescentes de 15 a 19 años $0,4 \%$ de alcohóli$\cos ^{11}$. En 1982 usando, en un estudio comparativo, las mismas definiciones de llorwitz, encontramos $2 \%$ de Alcohólicos en el nismo grupo de edad, es decir, un aumento de $400 \%^{12}$ dato que estaría testimoniando una situación muy grave que sería necesario corregir.

Para mejorar esta situación, es necesario pesquisar el beber anormal en sus comienzos y en sus formas disimuladas, donde hay mejores posibilidades terapéuticas. Si sólo captamos al joven cuando muestra signos de gran consumo o manifiestas complicaciones psiquicas o somátjcas, el pronóstico se ensombrece, pues si las complicaciones ocurren a temprana edad las consecuencias son más graves y menor la expectativa de vida ${ }^{14}$.

La búsqueda de formas clínicas manifiestas $y$ sobre todo disimuladas ha llevado a diversos 
investigadores, a buscar nuevos modos de conce. bir $y$ analizar la enfermedad y a proponer sus respectivas clasificaciones. En nuestro país, $\operatorname{Roa}^{1}$ ha establecido una clasificación que reproduce fielmente lo observado en nuestros pacientes. Asi, además del $\Lambda$ bstemio y Bebedor normal, reconoce a los Alcohólicos Normotimizantes, Tediofóbicos, Sintomáticos y Ocultos. Està concepción clínica del Alcoholismo es la que nos guía en nuestra investigación.

Para contribuir à un mejor diagnóstico, preverción y tratamiento de la enfermedad discniamos y realizamos un estudio cuyos objetivos especificos fueron conocer la prevalencia de] alcoholisno y otras formas de beber del adolescente en la población general e investigar la relación entre el hábito de beber y las variables socio demograficas y biológicas de sexo, edad. esirato sociocconómico, escolaridad y actividad.

\section{SUJETOS Y METODO}

El método de csta investigación, se ha publicado en detalle ${ }^{5}$; aqui expondremos sólo el resumen correspondiente.

l:1 universo fue defuido como la población de 15 a 19 años residentes en viviendas privadas de la zoma urbana de Santiago. El grupo que se iba a invesligar lue scleccionado mediante muestreo sistemático con arranque aleatorio y quedó conslituido por un tolal de 456 adolescentes encuestados. 229 hombres y 227 mujeres. El promedio de edad fue de 17.1 años y la distribución por sexoen cada edad. aproximadamente inual.

El estrato socineconómico fue definido según lat vivienda y el barrio. Lal distribución de los encuestados fue desigual, ya que $52,4 \%$ pertenecían al nivel medio-bajo y $21,3 \%$ al bajo.

En la muestra no labian analfabetos, todos alcanzaron algúr nivel de escolaridad, completono incompleto. Más de la mitad $(61,2 \%)$ cursaron hasta Enseñanza Media y $28.0 \%$ Básica.

Lid mayoril de los encuestados $(70,8 \%)$ se encuntraba en el momento de ser entrevistado, en una actividad estudiantil. En el grupo que no esludiaba, la sexta parte permanecía inactiva y el resto trabajaba con o sin remuncración.

Para recolectar la infortnación se diseñó un cuestionario, que incluye una entrevista clínica pautada para el diagnóstico de alcoholismo $y$ otros hábitos de beber, que habia sido probada $y$ validada anteriormente ${ }^{16}$.

Las entrevistas personales, fueror efectuadas por 200 estudiantes de medicina del último año de li carrera. preparados, entrenados especialmente y supervisados en terretio.

La informacion tue procesada en computador mediante un programa denominado Statistical Antalysis System.

Los sujetos fueron clasificados, de acuerdo con los conceptos de $\mathrm{Roa}^{1-2}$ en Alcohólicos Normatimizantes, Tediofóbicos, Sintomáticos y Ocultos. Nomales y Abstemios. En el curso de nuestras investigaciones se hizo evidente un gru. po intermedio que denominamos bebedores Farmacologicos, caracterizados por no necesitar habilualmente ef alcohol para enfrentar las vicisitudes de la vida diaria, pero que lo usan bus. cando sentirse relajados, agradados o compartien. do con un grupo: no se embriugan o sólo to hacen muy vcasionalmente pues beben en pequeñas cantidades.

\section{RESULTADOS}

La magnitud del alcoholismo y de los otros hábitos de beber en el adolescente. se muestra en la Tabla 1 .

Tahla 1 .

Habito di: Beber en Adolescentes

\begin{tabular}{|c|c|c|c|}
\hline Hébito & $\mathrm{No}$ & $\%$ & \\
\hline Abstemios & 164 & 36.0 & \\
\hline Normales & 237 & 51.9 & \\
\hline liarmacológicos & 13 & 2.3 & \\
\hline Alsohólicus & 42 & 9.2 & \\
\hline Vortnotimizantes & 34 & & 7.7 \\
\hline Tedtiofobicos & 6 & & 1.3 \\
\hline Sintom áticus & 1 & & 0.2 \\
\hline Oentess & $\mathbf{I}$ & & d. 2 \\
\hline TOTAL & 456 & 100.0 & \\
\hline
\end{tabular}

Se observa que de los 42 alcohólicos, 34 buscan un efecto Normotimizante, sienda más frecuentes las respuestas: "más seguridad (o confianza, o personalidad, o se desinhibe, o se le quita la verguienza)", o también: "más ánino (o energia, o alcgria)". Los alcohólicos Tediofóbicos son súlo 6, y el efecto está centrado principalmente en que: "se le acorta el tiempo". Encontramos solamente un alcohólico Sintomá tico y uno Oculto.

El grupo de bebedores Farmacológicos es pequeño, apruximadamente la mitad de bo encontrado en la población general. Las características de su beber son intermedias entre el normal y el alcohólico y corresponde más bien a un hábito propio del adulto ${ }^{2}$

Por otro lado, el $64 \%$ de los adolescentes beben alcohol, lo que cstá en el punto nedio de Ios resultados de distintos estudios ya mencionados, cuyos rangos van desde el $44 \%$ al $88 \%$ aproximadamente, rango muy auplio por el uso de muestras y definiciones distintas. 
La distribución del hảbito de beber según sexo, se presentá en la Tabla 2.

Tabla 2.

Hábitos de Beber $y$ Sexo

\begin{tabular}{|c|c|c|c|c|c|c|}
\hline \multirow[b]{2}{*}{ Ifibile } & \multicolumn{2}{|c|}{ Hombes } & \multicolumn{2}{|c|}{ Mujeres } & \multicolumn{2}{|c|}{ Tot:al } \\
\hline & NO & $\%$ & د: & $\%$ & $\mathrm{Na}$ & $\%$ \\
\hline Abstimius & 53 & 2.7 .1 & 111 & 49.0 & 164 & 36.0 \\
\hline Normales: & 130 & 56,8 & 107 & 47.1 & $23:$ & $\$ 1.9$ \\
\hline F¡tmacológltos & 12 & 5.2 & 1 & {$[7.4$} & 13 & 29 \\
\hline Ninholuas & 34 & 14.9 & a & 3.5 & 43 & $\$ .2$ \\
\hline Tot:al & 239 & $100 . i$ & 227 & Inte.(1) & 456 & $|D i\rangle$ \\
\hline
\end{tabular}

$\mathrm{X}^{2}=48.14 \quad \mathrm{P}<0.0 \mathrm{jus}$

Entre los hombres es más frecuente el alcoholismo $(14,9 \%)$ que entre tas mujeres $\{3.5 \%)$. conclusión que concuerda con otras investiga- ciones ya mencionadas.

Se observa que hay más bebedores normales entre los hombres y que las mujeres son principalmente abstemias. Las diferencias expuestas son estadisticamente significativas.

Para conocer la variación del hábito de beber según la edad, el estudio se hizo por grupos individuales de edades en años, lo que se denues. tra en la Tabla 3.

Entre los adolescentes de 17 años hay más alcohólicos, más bebedores normales y menos abstemios. Las diferencias no son estadísticamente significativas.

En la Tabla 4 se muestra la distribución de los adolescentes según estrato socio-económico, inferido de la calidad de la vivienda y del barrio. EI nivel 1 corresponde a los estratos alto, medio-alto $y$ medio; el nuvel 2 al estrato medio bajo y el nivel 3 al bajo.

Tabla 3.

Hábito de Bcber $y$ Fidad

\begin{tabular}{|c|c|c|c|c|c|c|}
\hline Hábito & $\begin{array}{c}15 \text { años } \\
\%, N=81\end{array}$ & $\begin{array}{c}16 \text { años } \\
\%_{Q}, N=90\end{array}$ & $\begin{array}{l}17 \text { งños } \\
\%, x=92\end{array}$ & $\begin{array}{l}\text { I B jos } \\
\%, N=98\end{array}$ & $\begin{array}{c}19 \text { años } \\
\% \mathrm{~N}=95\end{array}$ & $\begin{array}{c}\text { Tot:1 } \\
\% \mathrm{~N}=456\end{array}$ \\
\hline $\begin{array}{l}\text { Abstemios } \\
\text { Normales } \\
\text { Farmacológicos } \\
\text { Alcohólicos }\end{array}$ & $\begin{array}{r}50.7 \\
43.2 \\
1.2 \\
4.9\end{array}$ & $\begin{array}{r}36.7 \\
52.2 \\
3.3 \\
7.8\end{array}$ & $\begin{array}{r}27.2 \\
56.5 \\
3.3 \\
13.0\end{array}$ & $\begin{array}{r}31.6 \\
54.1 \\
4.1 \\
10.2\end{array}$ & $\begin{array}{r}35.8 \\
52.6 \\
2.1 \\
9.5\end{array}$ & $\begin{array}{r}36.0 \\
51.9 \\
2.9 \\
9.2\end{array}$ \\
\hline Total & 100.0 & 100.0 & 100.0 & 100.0 & 100.0 & 100.0 \\
\hline
\end{tabular}

Tabla 4.

lébito de Bcber y Estrato Socio-rconómico

\begin{tabular}{|c|c|c|c|c|c|c|c|c|}
\hline \multirow{2}{*}{ Hábito } & \multicolumn{2}{|c|}{1} & \multicolumn{2}{|c|}{$\underline{2}$} & \multicolumn{2}{|c|}{3} & \multicolumn{2}{|c|}{ Total } \\
\hline & No & $\%$ & $\mathrm{~N}^{\mathrm{O}}$ & $\%$ & No & $\%$ & No & $\%$ \\
\hline Abstemios & 28 & 28.9 & 94 & 39.3 & 42 & 35.0 & 164 & 36. \\
\hline Normales & 67 & 69.1 & 121 & 50.6 & 49 & 40.9 & 237 & 51.9 \\
\hline Farmacolögitos & ] & 1.0 & 3 & 3.4 & 4 & 3.3 & 13 & 2.9 \\
\hline Alcohólicos & 1 & ]. 0 & 16 & 6.7 & 25 & 20.8 & 42 & 9.2 \\
\hline Total & 97 & 100.0 & 239 & 100.0 & 320 & 100.0 & 456 & 100.0 \\
\hline
\end{tabular}

$\mathrm{X}^{2}=38.27 \mathrm{P}<0.005$

La tasa de alcoholismo, aumenta a medida que se baja de estrato, siendo mayor en el estrato bajo $(20,8 \%)$, lo inverso sucede con et beber normal, encontrando mayor frecuencia de ellos en los niveles más altos Las diferencias encontradas son estadisticamente significativas.

La relación con la escolaridad alcanzada se encuentra en la Tabla 5.

EJ Alcoholismo es más frecuente cuanto me- nor es el rivel educacional alcanzado, así el porcentaje es máximo en la escolaridad Básica (15\%). Lo inverso sucede con el beber normal. Las diferencias encontradas son estadísticamente significativas.

Dadas las caracteristicas anteriores parecio importante analizar el hábito de beber en relación a la condición de estudiante del adolescente, lo que se presenta en la Tabla 6 . 
Tabla 5

Hábito de Bebex y Fiscolaridad

\begin{tabular}{|c|c|c|c|c|c|c|c|c|}
\hline \multirow{2}{*}{ Hábito } & \multicolumn{2}{|c|}{ Básica } & \multicolumn{2}{|c|}{ Media } & \multicolumn{2}{|c|}{ Otros } & \multicolumn{2}{|c|}{ Total } \\
\hline & $\mathbf{N}^{\circ}$ & $\%$ & No & $\%$ & No & $\%$ & No & $\%$ \\
\hline Abstemios & 54 & 42.2 & 93 & 33.3 & 17 & 34.7 & 164 & 36.0 \\
\hline Normiles & 49 & $3 k .3$ & 158 & $\$ 6.6$ & 30 & 61.2 & 237 & 51.9 \\
\hline Farniacologions & s & 3.9 & 8 & 2.9 & 0 & 0.0 & 13 & 2.9 \\
\hline Aleohólicos & 20 & 15.6 & 20 & 7.2 & 2 & 4.1 & 42 & 9.2 \\
\hline Total & 128 & 100.0 & 279 & 100.0 & 47 & 100.0 & 456 & 100.0 \\
\hline
\end{tabular}

$\mathrm{X}^{2}=18.8 \mathrm{P}<0.01$

Tabla 6

Hábito de Beber y Condición de Estudiante.

\begin{tabular}{|c|c|c|c|c|c|c|}
\hline \multirow{2}{*}{ Hábito } & \multicolumn{2}{|c|}{ Estudiante } & \multicolumn{2}{|c|}{ No tistudiante } & \multicolumn{2}{|c|}{ Total } \\
\hline & No & $\%$ & $N^{O}$ & $\%$ & No & $\%$ \\
\hline Abstemios & 116 & 35.9 & 48 & 36.0 & 164 & 36.0 \\
\hline Normales & 176 & $\leq 4.5$ & 61 & 45.9 & 237 & 51.9 \\
\hline Parmaculógicos & 8 & 2.5 & 5 & 3.8 & 13 & 2.9 \\
\hline Alcohólicos & 23 & 7.1 & 19 & 14.3 & 42 & 9.2 \\
\hline Total & 232 & 100.0 & 133 & 100.0 & 456 & 100.0 \\
\hline
\end{tabular}

$\mathrm{X}^{2}=6.33 \quad \mathrm{P}<0.025$.

La frecuencia de Alcoliólicos era mayor en el grupo que no estudia, en cambio hay más Normales entre los estudiantes. Las diferencias son estadísticamente significativas sì se agrupan alcohólicos con bebedores farmacológicos por un lado y normales con abstemios por otro.

Un análisis más detallado permite obscrvar que, en el grupo do adolescentes que no estudia, el A coholismo cs más frecuente entre los cesan. tes que entre los que trabajan. Por otro lado, en los adolescentes que estudian, el Alcoholismo es más frecuente entre los alumnos de Enserianza Básica que en Mcdia y Superior.

\section{COMENTARIOS}

Siendo los alcohólicos 42 y la tasa de alcoholismo $9,2 \%$, en cifras absolutas tendríamos aproximadamente 40.000 alcuhólicos adolescentes de I5 a $J 9$ años en la Región Metropolitana. Esta cifra inesperadamente alta, se explica porque incluye alcoholismos graves junto con formas de comienzo y disinuladas.

La tasa de $9: 2 \%$. cercana a la del adulto joven $(12,4 \%)$, del adulto maduro $(14,8 \%)$ y del anciano $(13,8 \%)$, indicaría ciue en la actualidad, en la gran mayoría de los casos la enfermedad se establecería en esta etapa de la vida, a diferencia de lo que, al parecer, ocurría algunas décadas atrás, cuando la enfermedad habitualmente se iniciaba en la vida adulta. Si mediante un estudio de seguimiento se comprueba esta hipótesis, los programas preventıvos y terapéuticos deberán dirigirse preferentemente a este grupo de persofas y es posible suponer que los resultadus serán mejores.

La relación mujer-hombre alcohólico en ado. lescentes es de $1: 4$, proporción más estrecha que la encontrada en la población total 1:5, lo que sugiere que se estarian produciendo cambios en la actitud de la mujer respecto al alcohol.

En lo referente a alcoholismo y estrato social, at comparar estos resultados con los encontrados en la población general (que incluye todas las edades), obseryamos que las tasas son muy semejantes en el estrato bajo: $20.8 \%$ en adolescentes y $21.8 \%$ en la población general. En cambio, en el estrato medio-bajo hay clara diferencia en las tasas: $6,7 \%$ en adolescentes y $11,9 \%$ en la población general y también en el estrato agrupado de niveles más altos: en ađolescentes es de $1 \%$ y en la población general es de $5.9 \%$. Es decir, en el 
estrato bajo, la prevalercia del alcoholismo en adolescentes es semejante a la tasa enconlrada cuando se consideran todas las edades, to que no se da en las agrupaciones más altas. Esto indica que el nivel socio-económico es un factor impertante en la aparicion de la enfermedad.

Finalmente los resultados de este estudio sugieren la necesidad de conceder suma importancia no sólo a los programas de prevención del alcoholismo en las escuelas $y$ tas comanidades laborales, sino también entre los adolescentes que no estudian ni trabajan.

\section{RESUMEN}

Los aulores estudian el Alcoholismo y otrus híbitos de beber en la población adolescente do 15 a 19 años. utilizando el diagnóstico clínico a través de una entrevista individuat. La tasa de alcoholismo es altal y alcatza id un $9.2 \%$, siendo mús lrecuente el tipo Normotimizante

La tasa de alcohólicos adolescentes cs semejante a la de adultos y ancianos, cllo indicaría que la enfermedad se establecería en etapas tempranas de la vida, hipotesis a comprobar en un estudio de seguimiento.

La enfermedad afecta más a los humbres, siendo la razón alcoholismo femenino/masculino de $1 / 4$, más estrecha que en la población general, lo que podría señalar un cambio en el hábito de beber en las adolescentes mujercs.

El estrato social con más alcohólicos es el bajo, encontrando una proporción del $20.8 \%$.

El alcoholismo es más frecuente a menor nivel escolar y entre los jóvenes que no están estudiando.

Se puede cstablecer un perfil epidemiológico de los adolescentes de más alto ricsgo de enfernar, este es: adolescente hombre. de estrato social bajo, de escasa escolaridad, que no esludia y no trabaja.

Pensamos que las acciones de prevención debieran dirigirse principalmente a esta edad y en toda la comuridad.

\section{REFERENCIAS}

1. Roa, A.: "Los poderes del alcohol". Rev. de Psi-

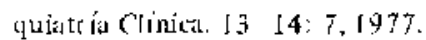

2. Pallavicini, J., Legerreta, A. y' otros: "Estudio Clínico y Lpjdemiológicu del Alcoholismo entendido conzo Apetencia Patológle ". Rev. de Psiquiatría Clínica. 20: 68.1983.

3. Motina, C. Mron cibia S. "vrros: "Djagnóstico de la situación del Aicotiolising y la Drogadicción". Informe Firal No 2. Serie de listudios N0 89. C.P F... P. Lo Barnechea, Oetubre, 1983 .

4. Cobrerd, F., Sahmon. C. $y$ orros: "Consumo de Brbidas Aloohóljeass en la Pohlación Lscolar ded Area Oecidente de Sintiano". Cuad. Med. Soi. 23: 5,1982 .

5. Repeten, A., Llomes, R. y otros: "Prevalencis do Ingesta Anormal de Bcbidals Aboholicas en Alunt. wos de Enseiranza Media de la Comuna de La Reinu". Cuad. Méd. Soc. $24: 84.1983$.

6. Florenzano. R., Madrid. $y$. y otros:" "Prevalencid y earacteristials del consumt? de iljotlas sustanciats quimicas en restudienter de linseñants Metia en Salntiage de Chile". Rev. Mcd. Chil., 109: 105!, 1981

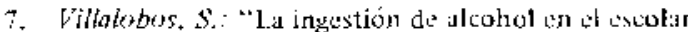
adolesecente". Cuad. Méd Soc. 21: 53. Marzo. 1980.

8. Ingchs Jents't, O., Rud, M.G.: "Changes in the use of Drups, Tabacco, and Rlushol among Norwegian youth frotr 1968 1979". J. Drug Issucs 40. 405. 1980.

9. Lemperiere, J., Ades, G. y otros: Les eonduites alcooliques de l'adolescent ct de l'adulte jane, a propos de 1 is observitions". Anuales Méd. Prychol. 140: 1003. Nov. 1982

10. Migucz, H.A.: "Previlenteia de niveles de lngestion de Akohol an Costil Rica". Bol. 01. Sanit. Panam. 95: $451,1983$.

11. Horwisz. J., Muñoz, L. y otros: "Investigaciones epidemiológicas acerca de la morbilidad mental en Chile. Rev. S.N.S. 3: 277, 1958.

12. Pallavicini, J, Lezarreta, A. y otros: "listudio usmparutuvo sobre Akohulisino y otros híbitos de beber, Santiago 1958 1982". Rev. de Psiquiatr f́a Clínica. 20: $48,1983$.

13. Roa, A.: "Ias toansfomacionts de los cuadros mentiles las ćpocas históricas" Médico 1:2, 1976.

14. Nawillan, P. Vargas, $\mathrm{S}$.

14. Noveillan. P. Vargas, $S$. "Expectutiva de vida de. bebedar problema en Sintiago. Chiłe Jll. Estima. ción de tasas específicas de mortalidad". Bot. Of. Sanjt. Panam. 96: 334, 1984.

15. Pallovicini, J., Legarreta, A. y orros; "Caracteristicas y Metodologia de la investigación Clínica $y$ Epidemiológias sobre Akoholismo y otras Apetencias". Rev. de Psiquiatr ía Clínica. 20: 21, 1983.

16. Pallavicini, J., Legorre'ta, A. y otros: "Vatidación do un Instrumento para el Disgnóstico dc Alcoholismo en la Población". Rev. de Psicutiatria Clínica. 20: $36,1983$. 